\title{
EXACT WAVE FUNCTIONS FOR GENERALIZED HARMONIC OSCILLATORS
}

\author{
NATHAN LANFEAR, RAQUEL M. LÓPEZ, AND SERGEI K. SUSLOV
}

\begin{abstract}
We transform the time-dependent Schrödinger equation for the most general variable quadratic Hamiltonians into a standard autonomous form. As a result, the time-evolution of exact wave functions of generalized harmonic oscillators is determined in terms of solutions of certain Ermakov and Riccati-type systems. In addition, we show that the classical Arnold transformation is naturally connected with Ehrenfest's theorem for generalized harmonic oscillators.
\end{abstract}

\section{INTRODUCTION}

Quantum systems with variable quadratic Hamiltonians are called the generalized harmonic oscillators (see [4], 9], [14], [15], [19], 222, [26], [33], [35], 37], [40], 54], [55], [56] and references therein). These systems have attracted substantial attention over the years because of their great importance in many advanced quantum problems. Examples are coherent states and uncertainty relations, Berry's phase, quantization of mechanical systems and Hamiltonian cosmology. More applications include, but are not limited to charged particle traps and motion in uniform magnetic fields, molecular spectroscopy and polyatomic molecules in varying external fields, crystals through which an electron is passing and exciting the oscillator modes, and other mode interactions with external fields. Quadratic Hamiltonians have particular applications in quantum electrodynamics because the electromagnetic field can be represented as a set of forced harmonic oscillators [22].

A goal of this Letter is to construct exact wave functions for generalized (driven) harmonic oscillators [4], 7], 26], 333], 35], [54], 55], in terms of Hermite polynomials by transforming the time-dependent Schrödinger equation into an autonomous form [56]. The relationship with certain Ermakov and Riccati-type systems, which seem are missing in the available literature in general, are investigated. A group theoretical approach to a similar class of partial differential equations is discussed in Refs. [1], [6], [12], [24], [42], [45] (see also [48], 49] and references therein). Some applications to the nonlinear Schrödinger equation can also be found in Refs. [13], [27], [28], [29], [30], [44, [47] and [51].

\section{Transforming Generalized Harmonic Oscillators into Autonomous Form}

We consider the one-dimensional time-dependent Schrödinger equation

$$
i \frac{\partial \psi}{\partial t}=H \psi
$$

Date: November 7, 2018.

1991 Mathematics Subject Classification. Primary 81Q05, 35C05. Secondary 42A38.

Key words and phrases. The time-dependent Schrödinger equation, generalized harmonic oscillators, Green's function, propagator, Ermakov-type invariant and Pinney-type solution, Ehrenfest theorem, Arnold transformation. 
where the variable Hamiltonian $H=Q(p, x)$ is an arbitrary quadratic of two operators $p=-i \partial / \partial x$ and $x$, namely,

$$
i \psi_{t}=-a(t) \psi_{x x}+b(t) x^{2} \psi-i c(t) x \psi_{x}-i d(t) \psi-f(t) x \psi+i g(t) \psi_{x},
$$

$(a, b, c, d, f$ and $g$ are suitable real-valued functions of time only). We shall refer to these quantum systems as the generalized (driven) harmonic oscillators. Some examples, a general approach and known elementary solutions can be found in Refs. [7], [8], [9], [10], [14], [20], [21], [22], [35], [36], [39], [46], [54] and [55]. In addition, a case related to Airy functions is discussed in [32] and Ref. [11] deals with another special case of transcendental solutions.

The following is our first result.

Lemma 1. The substitution

$$
\psi=\frac{e^{i\left(\alpha(t) x^{2}+\delta(t) x+\kappa(t)\right)}}{\sqrt{\mu(t)}} \chi(\xi, \tau), \quad \xi=\beta(t) x+\varepsilon(t), \quad \tau=\gamma(t)
$$

transforms the non-autonomous and inhomogeneous Schrödinger equation (2.2) into the autonomous form

provided that

$$
-i \chi_{\tau}=-\chi_{\xi \xi}+c_{0} \xi^{2} \chi \quad\left(c_{0}=0,1\right)
$$

$$
\begin{gathered}
\frac{d \alpha}{d t}+b+2 c \alpha+4 a \alpha^{2}=c_{0} a \beta^{4} \\
\frac{d \beta}{d t}+(c+4 a \alpha) \beta=0, \\
\frac{d \gamma}{d t}+a \beta^{2}=0
\end{gathered}
$$

and

$$
\begin{gathered}
\frac{d \delta}{d t}+(c+4 a \alpha) \delta=f+2 g \alpha+2 c_{0} a \beta^{3} \varepsilon, \\
\frac{d \varepsilon}{d t}=(g-2 a \delta) \beta, \\
\frac{d \kappa}{d t}=g \delta-a \delta^{2}+c_{0} a \beta^{2} \varepsilon^{2} .
\end{gathered}
$$

Here

$$
\alpha=\frac{1}{4 a} \frac{\mu^{\prime}}{\mu}-\frac{d}{2 a}
$$

Proof. Differentiating $\psi=\mu^{-1 / 2}(t) e^{i S(x, t)} \chi(\xi, \tau)$ with $S=\alpha(t) x^{2}+\delta(t) x+\kappa(t), \xi=\beta(t) x+\varepsilon(t)$ and $\tau=\gamma(t)$ yields

$$
\begin{gathered}
i e^{-i S} \psi_{t}=\frac{1}{\sqrt{\mu}}\left[-\left(\alpha^{\prime} x^{2}+\delta^{\prime} x+\kappa^{\prime}\right) \chi+i\left(\left(\beta^{\prime} x+\varepsilon^{\prime}\right) \chi_{\xi}+\gamma^{\prime} \chi_{\tau}-\frac{\mu^{\prime}}{2 \mu} \chi\right)\right] \\
e^{-i S} \psi_{x}=\frac{1}{\sqrt{\mu}}\left[i(2 \alpha x+\delta) \chi+\beta \chi_{\xi}\right]
\end{gathered}
$$

and

$$
e^{-i S} \psi_{x x}=\frac{1}{\sqrt{\mu}}\left[\left(2 i \alpha-(2 \alpha x+\delta)^{2}\right) \chi+2 i(2 \alpha x+\delta) \beta \chi_{\xi}+\beta^{2} \chi_{\xi \xi}\right] \text {. }
$$


Substituting into

$$
\begin{aligned}
i \psi_{t}= & -a \psi_{x x}+\left(b-c_{0} a \beta^{4}\right) x^{2} \psi-i c x \psi_{x}-i d \psi \\
& -\left(f+2 c_{0} a \beta^{3} \varepsilon\right) x \psi+i g \psi_{x}-c_{0} a \beta^{2} \varepsilon^{2} \psi+c_{0} a \beta^{2} \varepsilon^{2} \xi^{2} \psi
\end{aligned}
$$

and using system (2.5)-(2.10), results in Eq. (2.4). Further computational details are left to the reader.

Our transformation (2.3) provides a new interpretation to system (2.5)-(2.10) originally derived in Ref. [7] when $c_{0}=0$ by integrating the corresponding Schrödinger equation via the Green function method (see also [50] for an eigenfunction expansion). Here, we discuss the case $c_{0} \neq 0$ as its natural extension.

The substitution (2.11), which has been already used in [7], appears here from a new "transformation perspective". It now reduces the inhomogeneous equation (2.5) to the second order ordinary differential equation

$$
\mu^{\prime \prime}-\tau(t) \mu^{\prime}+4 \sigma(t) \mu=c_{0}(2 a)^{2} \beta^{4} \mu,
$$

that has the familiar time-varying coefficients

$$
\tau(t)=\frac{a^{\prime}}{a}-2 c+4 d, \quad \sigma(t)=a b-c d+d^{2}+\frac{d}{2}\left(\frac{a^{\prime}}{a}-\frac{d^{\prime}}{d}\right) .
$$

(The reader should be convinced that this derivation is rather straightforward.)

When $c_{0}=0$, equation (2.5) is called the Riccati nonlinear differential equation [52], 53]; consequently, the system (2.5)-(2.10) shall be referred to as a Riccati-type system. (Similar terminology is used in [49] for the corresponding parabolic equation.) Now if $c_{0}=1$, equation (2.16) can be reduced to a generalized version of the Ermakov nonlinear differential equation (5.1) (see, for example, [9], 18], 34], [50] and references therein regarding Ermakov's equation) and we shall refer to the corresponding system (2.5)-(2.10) with $c_{0} \neq 0$ as an Ermakov-type system.

\section{Green's Function and Wavefunctions}

Two particular solutions of the time-dependent Schrödinger equation (2.2) are useful in physical applications. Using standard oscillator wave functions for equation (2.4) when $c_{0}=1$ (for example, [23], [31] and/or [41]) results in the solution

$$
\psi_{n}(x, t)=\frac{e^{i\left(\alpha x^{2}+\delta x+\kappa\right)+i(2 n+1) \gamma}}{\sqrt{2^{n} n ! \mu \sqrt{\pi}}} e^{-(\beta x+\varepsilon)^{2} / 2} H_{n}(\beta x+\varepsilon),
$$

where $H_{n}(x)$ are the Hermite polynomials [43], provided that the solution of the Ermakov-type system (2.5) -(2.10) is available.

The Green function of generalized harmonic oscillators has been constructed in the following fashion in Ref. [7]:

$$
G(x, y, t)=\frac{1}{\sqrt{2 \pi i \mu_{0}(t)}} \exp \left[i\left(\alpha_{0}(t) x^{2}+\beta_{0}(t) x y+\gamma_{0}(t) y^{2}+\delta_{0}(t) x+\varepsilon_{0}(t) y+\kappa_{0}(t)\right)\right]
$$


The time-dependent coefficients $\alpha_{0}, \beta_{0}, \gamma_{0}, \delta_{0}, \varepsilon_{0}, \kappa_{0}$ satisfy the Riccati-type system (2.5)-(2.10) $\left(c_{0}=0\right)$ and are given as follows [7], [46], [50]:

$$
\begin{aligned}
& \alpha_{0}(t)=\frac{1}{4 a(t)} \frac{\mu_{0}^{\prime}(t)}{\mu_{0}(t)}-\frac{d(t)}{2 a(t)} \\
& \beta_{0}(t)=-\frac{\lambda(t)}{\mu_{0}(t)}, \quad \lambda(t)=\exp \left(-\int_{0}^{t}(c(s)-2 d(s)) d s\right) \\
& \gamma_{0}(t)=\frac{1}{2 \mu_{1}(0)} \frac{\mu_{1}(t)}{\mu_{0}(t)}+\frac{d(0)}{2 a(0)}
\end{aligned}
$$

and

$$
\begin{aligned}
& \delta_{0}(t)= \frac{\lambda(t)}{\mu_{0}(t)} \int_{0}^{t}\left[\left(f(s)-\frac{d(s)}{a(s)} g(s)\right) \mu_{0}(s)+\frac{g(s)}{2 a(s)} \mu_{0}^{\prime}(s)\right] \frac{d s}{\lambda(s)} \\
& \begin{aligned}
\varepsilon_{0}(t)= & -\frac{2 a(t) \lambda(t)}{\mu_{0}^{\prime}(t)} \delta_{0}(t)+8 \int_{0}^{t} \frac{a(s) \sigma(s) \lambda(s)}{\left(\mu_{0}^{\prime}(s)\right)^{2}}\left(\mu_{0}(s) \delta_{0}(s)\right) d s \\
& +2 \int_{0}^{t} \frac{a(s) \lambda(s)}{\mu_{0}^{\prime}(s)}\left(f(s)-\frac{d(s)}{a(s)} g(s)\right) d s \\
\kappa_{0}(t)= & \frac{a(t) \mu_{0}(t)}{\mu_{0}^{\prime}(t)} \delta_{0}^{2}(t)-4 \int_{0}^{t} \frac{a(s) \sigma(s)}{\left(\mu_{0}^{\prime}(s)\right)^{2}}\left(\mu_{0}(s) \delta_{0}(s)\right)^{2} d s \\
& -2 \int_{0}^{t} \frac{a(s)}{\mu_{0}^{\prime}(s)}\left(\mu_{0}(s) \delta_{0}(s)\right)\left(f(s)-\frac{d(s)}{a(s)} g(s)\right) d s
\end{aligned}
\end{aligned}
$$

$\left(\delta_{0}(0)=-\varepsilon_{0}(0)=g(0) /(2 a(0))\right.$ and $\left.\kappa_{0}(0)=0\right)$ provided that $\mu_{0}$ and $\mu_{1}$ are standard solutions of equation (2.16) with $c_{0}=0$ corresponding to the initial conditions $\mu_{0}(0)=0, \mu_{0}^{\prime}(0)=2 a(0) \neq 0$ and $\mu_{1}(0) \neq 0, \mu_{1}^{\prime}(0)=0$. (Proofs of these facts are outlined in Refs. [7], [1] and [46]. See also important previous works [15], [37], [54], [55], [56] and references therein for more details.)

Hence, the corresponding Cauchy initial value problem can be solved (formally) by the superposition principle:

$$
\psi(x, t)=\int_{-\infty}^{\infty} G(x, y, t) \psi(y, 0) d y
$$

for some suitable initial data $\psi(x, 0)=\varphi(x)$ (see Refs. [7], [46] and [50] for further details).

In particular, using the wave functions (3.1) we get the integral

$$
\psi_{n}(x, t)=\int_{-\infty}^{\infty} G(x, y, t) \psi_{n}(y, 0) d y
$$

and this can be evaluated by

$$
\begin{aligned}
& \int_{-\infty}^{\infty} e^{-\lambda^{2}(x-y)^{2}} H_{n}(a y) d y \\
& =\frac{\sqrt{\pi}}{\lambda^{n+1}}\left(\lambda^{2}-a^{2}\right)^{n / 2} H_{n}\left(\frac{\lambda a x}{\left(\lambda^{2}-a^{2}\right)^{1 / 2}}\right), \quad \operatorname{Re} \lambda^{2}>0
\end{aligned}
$$

which is an integral transform equivalent to Eq. (30) on page 195 of Vol. 2 of Ref. [16] (the Gauss transform of Hermite polynomials), or Eq. (17) on page 290 of Vol. 2 of Ref. [17. 


\section{Solution to Ermakov-Type System}

As shown in the previous section, the time evolution of the wave functions (3.1) is determined in terms of the solution to the initial value problem for the Ermakov-type system. In this section, formulas (3.1) $-(3.2)$ and (3.10) $-(3.11)$ shall be used in order to solve the general system (2.5) $-(2.10)$ when $c_{0} \neq 0$ along with the uniqueness property of the Cauchy initial value problem. At this point, we must remind the reader how to handle the special case $c_{0}=0$ considered in [46].

Lemma 2. The solution of the Riccati-type system (2.5)-2.10) $\left(c_{0}=0\right)$ is given by

$$
\begin{aligned}
& \mu(t)=2 \mu(0) \mu_{0}(t)\left(\alpha(0)+\gamma_{0}(t)\right), \\
& \alpha(t)=\alpha_{0}(t)-\frac{\beta_{0}^{2}(t)}{4\left(\alpha(0)+\gamma_{0}(t)\right)}, \\
& \beta(t)=-\frac{\beta(0) \beta_{0}(t)}{2\left(\alpha(0)+\gamma_{0}(t)\right)}=\frac{\beta(0) \mu(0)}{\mu(t)} \lambda(t), \\
& \gamma(t)=\gamma(0)-\frac{\beta^{2}(0)}{4\left(\alpha(0)+\gamma_{0}(t)\right)}
\end{aligned}
$$

and

$$
\begin{aligned}
& \delta(t)=\delta_{0}(t)-\frac{\beta_{0}(t)\left(\delta(0)+\varepsilon_{0}(t)\right)}{2\left(\alpha(0)+\gamma_{0}(t)\right)}, \\
& \varepsilon(t)=\varepsilon(0)-\frac{\beta(0)\left(\delta(0)+\varepsilon_{0}(t)\right)}{2\left(\alpha(0)+\gamma_{0}(t)\right)}, \\
& \kappa(t)=\kappa(0)+\kappa_{0}(t)-\frac{\left(\delta(0)+\varepsilon_{0}(t)\right)^{2}}{4\left(\alpha(0)+\gamma_{0}(t)\right)}
\end{aligned}
$$

in terms of the fundamental solution (3.3)-(3.8) subject to the arbitrary initial data $\mu(0), \alpha(0)$, $\beta(0) \neq 0, \gamma(0), \delta(0), \varepsilon(0), \kappa(0)$.

This solution can be verified by a direct substitution and/or by an integral evaluation. This result can also be thought of as a nonlinear superposition principle for the Riccati-type system and the continuity with respect to initial data holds [46].

Hence, the solution (4.1) -(4.7) implies the following asymptotics established in [46]:

$$
\begin{aligned}
& \alpha_{0}(t)=\frac{1}{4 a(0) t}-\frac{c(0)}{4 a(0)}-\frac{a^{\prime}(0)}{8 a^{2}(0)}+\mathcal{O}(t), \\
& \beta_{0}(t)=-\frac{1}{2 a(0) t}+\frac{a^{\prime}(0)}{4 a^{2}(0)}+\mathcal{O}(t), \\
& \gamma_{0}(t)=\frac{1}{4 a(0) t}+\frac{c(0)}{4 a(0)}-\frac{a^{\prime}(0)}{8 a^{2}(0)}+\mathcal{O}(t), \\
& \delta_{0}(t)=\frac{g(0)}{2 a(0)}+\mathcal{O}(t), \quad \varepsilon_{0}(t)=-\frac{g(0)}{2 a(0)}+\mathcal{O}(t), \\
& \kappa_{0}(t)=\mathcal{O}(t)
\end{aligned}
$$


as $t \rightarrow 0$ for sufficiently smooth coefficients of the original Schrödinger equation (2.2). Therefore,

$$
\begin{aligned}
G(x, y, t) \sim & \frac{1}{\sqrt{2 \pi i a(0) t}} \exp \left[i \frac{(x-y)^{2}}{4 a(0) t}\right] \\
& \times \exp \left[-i\left(\frac{a^{\prime}(0)}{8 a^{2}(0)}(x-y)^{2}+\frac{c(0)}{4 a(0)}\left(x^{2}-y^{2}\right)-\frac{g(0)}{2 a(0)}(x-y)\right)\right]
\end{aligned}
$$

as $t \rightarrow 0$ (where $f \sim g$ as $t \rightarrow 0$, if $\lim _{t \rightarrow 0}(f / g)=1$ ). This corrects an errata in Ref. [7] .

Finally, we present the extension to a general case when $c_{0} \neq 0$. Our main result is the following.

Lemma 3. The solution of the Ermakov-type system (2.5)- (2.10) when $c_{0}=1(\neq 0)$ is given by

$$
\begin{aligned}
& \mu=\mu(0) \mu_{0} \sqrt{\beta^{4}(0)+4\left(\alpha(0)+\gamma_{0}\right)^{2}}, \\
& \alpha=\alpha_{0}-\beta_{0}^{2} \frac{\alpha(0)+\gamma_{0}}{\beta^{4}(0)+4\left(\alpha(0)+\gamma_{0}\right)^{2}}, \\
& \beta=-\frac{\beta(0) \beta_{0}}{\sqrt{\beta^{4}(0)+4\left(\alpha(0)+\gamma_{0}\right)^{2}}}=\frac{\beta(0) \mu(0)}{\mu(t)} \lambda(t), \\
& \gamma=\gamma(0)-\frac{1}{2} \arctan \frac{\beta^{2}(0)}{2\left(\alpha(0)+\gamma_{0}\right)}, \quad a(0)>0
\end{aligned}
$$

and

$$
\begin{aligned}
\delta= & \delta_{0}-\beta_{0} \frac{\varepsilon(0) \beta^{3}(0)+2\left(\alpha(0)+\gamma_{0}\right)\left(\delta(0)+\varepsilon_{0}\right)}{\beta^{4}(0)+4\left(\alpha(0)+\gamma_{0}\right)^{2}}, \\
\varepsilon= & \frac{2 \varepsilon(0)\left(\alpha(0)+\gamma_{0}\right)-\beta(0)\left(\delta(0)+\varepsilon_{0}\right)}{\sqrt{\beta^{4}(0)+4\left(\alpha(0)+\gamma_{0}\right)^{2}}} \\
\kappa= & \kappa(0)+\kappa_{0}-\varepsilon(0) \beta^{3}(0) \frac{\delta(0)+\varepsilon_{0}}{\beta^{4}(0)+4\left(\alpha(0)+\gamma_{0}\right)^{2}} \\
& +\left(\alpha(0)+\gamma_{0}\right) \frac{\varepsilon^{2}(0) \beta^{2}(0)-\left(\delta(0)+\varepsilon_{0}\right)^{2}}{\beta^{4}(0)+4\left(\alpha(0)+\gamma_{0}\right)^{2}}
\end{aligned}
$$

in terms of the fundamental solution (3.3)-(3.8) subject to the arbitrary initial data $\mu(0), \alpha(0)$, $\beta(0) \neq 0, \gamma(0), \delta(0), \varepsilon(0), \kappa(0)$.

Following are the steps to the sketch of the proof. Evaluate the integral (3.10) with the help of (3.11) by completing the square and simplify. Use the uniqueness property of the Cauchy initial value problem. One can also verify our solution by a direct substitution into the system (2.5) -(2.10) when $c_{0}=1$. These elementary but rather tedious calculations are left to the reader (the use of a computer algebra system is helpful at certain steps).

Furthermore, the asymptotics (4.8)-(4.12) together with our formulas (4.14)-(4.20) result in the continuity with respect to initial data:

$$
\lim _{t \rightarrow 0^{+}} \mu(t)=\mu(0), \quad \lim _{t \rightarrow 0^{+}} \alpha(t)=\alpha(0), \quad \text { etc. }
$$


Thus the transformation property (4.14)-(4.20) allows us to find a solution of the initial value problem in terms of the fundamental solution (3.3)-(3.8) and it may be referred to as a nonlinear superposition principle for the Ermakov-type system.

\section{Solution of the Ermakov-type Equation}

Starting from (2.16) $-(2.17)$ when $c_{0}=1$, and using (4.16) we arrive at

$$
\mu^{\prime \prime}-\tau(t) \mu^{\prime}+4 \sigma(t) \mu=(2 a)^{2}(\beta(0) \mu(0) \lambda)^{4} \mu^{-3},
$$

which is a familiar Ermakov-type equation (see [5], [9], [18], [34], [50], [56] and references therein). Then our formula (4.14) leads to the representation

$$
\left(\frac{\mu(t)}{\mu(0)}\right)^{2}=\beta^{4}(0) \mu_{0}^{2}(t)+\left(\frac{\mu_{1}(t)}{\mu_{1}(0)}+\frac{\mu^{\prime}(0)}{2 \mu(0)} \frac{\mu_{0}(t)}{a(0)}\right)^{2}
$$

given in terms of standard solutions $\mu_{0}$ and $\mu_{1}$ of the linear characteristic equation (2.16) when $c_{0}=0$. Further details on this Pinney-type solution and the corresponding Ermakov-type invariant are left to the reader (see also [5] and [50]).

\section{Ehrenfest Theorem Transformations}

By introducing an expectation value of the coordinate operator in the following form

$$
\bar{x}=\frac{\langle x\rangle}{\langle 1\rangle}=\frac{\langle\psi, x \psi\rangle}{\langle\psi, \psi\rangle},
$$

one can derive Ehrenfest's theorem for the generalized (driven) harmonic oscillators (see, for example, [8] and [9]). Then the following classical equation of motion of the parametric driven oscillator holds

$$
\frac{d^{2} \bar{x}}{d t^{2}}-\frac{a^{\prime}}{a} \frac{d \bar{x}}{d t}+\left(4 a b-c^{2}+c \frac{a^{\prime}}{a}-c^{\prime}\right) \bar{x}=2 a f-g^{\prime}+g \frac{a^{\prime}}{a}-c g .
$$

The transformation of the expectation values

$$
\bar{\xi}=\beta \bar{x}+\varepsilon, \quad \bar{\xi}=\langle\chi, \xi \chi\rangle \quad \text { with } \quad\langle\chi, \chi\rangle=1,
$$

corresponding to our Lemma 1, converts (6.2) into the simplest equation of motion of the free particle and/or harmonic oscillator:

$$
\frac{d^{2} \bar{\xi}}{d \tau^{2}}+4 c_{0} \bar{\xi}=0 \quad\left(c_{0}=0,1\right)
$$

(This can be verified by a direct calculation.)

Remark 1. An exact transformation of a linear second-order differential equation into the equation of motion of free particle was discussed by Arnold [3]. An extension of the later to the case of the time-dependent Schrödinger equation had been considered, for example, in Ref. [56] and recently it has been reproduced as the quantum Arnold transformation in [2] and [25] (see also [1], [6], [12], [24], [30], [42], 45] and [51] for similar transformations of nonlinear Schrödinger and other equations of mathematical physics). We elaborate on a relation of the quantum Arnold transformation for the generalized (driven) harmonic oscillators with a Riccati-type system when $c_{0}=0$ (transformation to the free particle) and consider an extension of this transformation (in terms of solutions of the 
corresponding Ermakov-type system) to the case $c_{0}=1$ (transformation to the classical harmonic oscillator [56]).

\section{Conclusion}

In this Letter, we have determined the time evolution of the wave functions of generalized (driven) harmonic oscillators (3.1), known for their great importance in many advanced quantum problems [22], in terms of the solution to the Ermakov-type system (2.5)-(2.10) by means of a variant of the nonlinear superposition principle (4.14)-(4.20). Moreover, the classical Arnold transformation is related to Ehrenfest's theorem. Numerous examples, the corresponding coherent states, dynamic invariants, eigenfunction expansions and transition amplitudes [15], [32], [33], [35], [37], [38], [50] will be discussed elsewhere.

Acknowledgments. We thank Professor Carlos Castillo-Chávez and Professor Vladimir I. Man'ko for support, valuable discussions and encouragement. The authors are indebted to Professor Francisco F. López-Ruiz for kindly pointing out the papers [2] and 25] to our attention and for valuable discussions. We are grateful to the organizers of the $12^{\text {th }}$ ICSSUR (Foz do Iguaçu, Brazil, May 0206, 2011) for their hospitality and an opportunity to present the results of this work. This research is supported in part by the National Science Foundation-Enhancing the Mathematical Sciences Workforce in the 21st Century (EMSW21), award \# 0838705; the Alfred P. Sloan Foundation-Sloan National Pipeline Program in the Mathematical and Statistical Sciences, award \# LTR 05/19/09; and the National Security Agency-Mathematical \& Theoretical Biology Institute - Research program for Undergraduates; award \# H98230-09-1-0104.

\section{REFERENCES}

[1] M. Ablowitz and P. A. Clarkson, Solitons, Nonlinear Evolution Equations and Inverse Scattering, Cambridge Univ. Press, Cambridge, 1991.

[2] V. Aldaya, F. Cossio, J. Guerrero and F. F. López-Ruiz, The quantum Arnold transformation, J. Phys. A: Math. Theor. 44 (2011), 065302.

[3] V. I. Arnold, Geometrical Methods in the Theory of Ordinary Differential Equations, Springer-Verlag, Berlin, Cambridge, 1998.

[4] M. V. Berry, Classical adiabatic angles and quantum adiabatic phase, J. Phys. A: Math. Gen. 18 (1985) \# 1, $15-27$

[5] J. F. Cariñena and J. de Lucas, A nonlinear superposition rule for solutions of the Milne-Pinney equation, Phys. Lett. A 372 (2008) \#33, 5385-5389.

[6] P. A. Clarkson, Painlevé analysis for the damped, driven nonlinear Schrödinger equation, Proc. Roy. Soc. Edin., 109A (1988), 109-126.

[7] R. Cordero-Soto, R. M. Lopez, E. Suazo and S. K. Suslov, Propagator of a charged particle with a spin in uniform magnetic and perpendicular electric fields, Lett. Math. Phys. 84 (2008) \#2-3, 159-178.

[8] R. Cordero-Soto, E. Suazo and S. K. Suslov, Models of damped oscillators in quantum mechanics, Journal of Physical Mathematics, 1 (2009), S090603 (16 pages).

[9] R. Cordero-Soto, E. Suazo and S. K. Suslov, Quantum integrals of motion for variable quadratic Hamiltonians, Ann. Phys. 325 (2010) \#9, 1884-1912.

[10] R. Cordero-Soto and S. K. Suslov, Time reversal for modified oscillators, Theoretical and Mathematical Physics 162 (2010) \#3, 286-316; see also arXiv:0808.3149v9 [math-ph] 8 Mar 2009.

[11] R. Cordero-Soto and S. K. Suslov, The degenerate parametric oscillator and Ince's equation, J. Phys. A: Math. Theor. 44 (2011) \#1, 015101 (9 pages); see also arXiv:1006.3362v3 [math-ph] 2 Jul 2010.

[12] M. Craddock, Fundamental solutions, transition densities and the integration of Lie symmetries, J. Diff. Eqs. 207 (2009) \#6, 2538-2560. 
[13] F. Dalfovo, S. Giorgini, L. P. Pitaevskii and S. Stringari, Theory of Bose-Einstein condensation in trapped gases, Rev. Mod. Phys. 71 (1999), 463-512.

[14] V. V. Dodonov, I. A. Malkin and V. I. Man'ko, Integrals of motion, Green functions, and coherent states of dynamical systems, Int. J. Theor. Phys. 14 (1975) \# 1, 37-54.

[15] V. V. Dodonov and V. I. Man'ko, Invariants and correlated states of nonstationary quantum systems, in: Invariants and the Evolution of Nonstationary Quantum Systems, Proceedings of Lebedev Physics Institute, vol. 183, pp. 71-181, Nauka, Moscow, 1987 [in Russian]; English translation published by Nova Science, Commack, New York, 1989, pp. 103-261.

[16] A. Erdélyi, Higher Transcendental Functions, Vols. I-III, A. Erdélyi, ed., McGraw-Hill, 1953.

[17] A. Erdélyi, Tables of Integral Transforms, Vols. I-II, A. Erdélyi, ed., McGraw-Hill, 1954.

[18] V. P. Ermakov, Second-order differential equations. Conditions of complete integrability, Universita Izvestia Kiev, Series III 9 (1880), 1-25; see also Appl. Anal. Discrete Math. 2 (2008) \#2, 123-145 for English translation of Ermakov's original paper.

[19] L. D. Faddeev, Feynman integrals for singular Lagrangians, Theoretical and Mathematical Physics 1 (1969) \#1, 3-18 [in Russian].

[20] R. P. Feynman, The Principle of Least Action in Quantum Mechanics, Ph. D. thesis, Princeton University, 1942; reprinted in: "Feynman's Thesis - A New Approach to Quantum Theory", (L. M. Brown, Editor), World Scientific Publishers, Singapore, 2005, pp. 1-69.

[21] R. P. Feynman, Space-time approach to non-relativistic quantum mechanics, Rev. Mod. Phys. 20 (1948) \# 2, 367-387; reprinted in: "Feynman's Thesis - A New Approach to Quantum Theory", (L. M. Brown, Editor), World Scientific Publishers, Singapore, 2005, pp. 71-112.

[22] R. P. Feynman and A. R. Hibbs, Quantum Mechanics and Path Integrals, McGraw-Hill, New York, 1965.

[23] S. Flügge, Practical Quantum Mechanics, Springer-Verlag, Berlin, 1999.

[24] L. Gagnon and P. Winternitz, Symmetry classes of variable coefficient nonlinear Schrödinger equations, J. Phys. A: Math. Gen. 26 (1993), 7061-7076.

[25] J. Guerrero, and F. F. López-Ruiz, V. Aldaya and F. Cossio, Harmonic states for the free particle, arXiv:1010.5525v3 [quant-ph] 1 Jul 2011.

[26] J. H. Hannay, Angle variable holonomy in adiabatic excursion of an integrable Hamiltonian, J. Phys. A: Math. Gen 18 (1985) \# 2, 221-230.

[27] Yu. Kagan, E. L. Surkov and G. V. Shlyapnikov, Evolution of Bose-condensed gas under variations of the confining potential, Phys. Rev. A 54 (1996) \#3, R1753-R1756.

[28] Yu. Kagan, E. L. Surkov and G. V. Shlyapnikov, Evolution of Bose gas in anisotropic time-dependent traps, Phys. Rev. A 55 (1997) \#1, R18-R21.

[29] Yu. S. Kivshar, T. J. Alexander and S. K. Turitsyn, Nonlinear modes of a macroscopic quantum oscillator, Phys. Lett. A 278 (2001) \#1, 225-230.

[30] A. Kundu, Integrable nonautonomous Schrödinger equations are equivalent to the standard autonomous equation, Phys. Rev. E 79 (2009), 015601(R) (4 pages).

[31] L. D. Landau and E. M. Lifshitz, Quantum Mechanics: Nonrelativistic Theory, Pergamon Press, Oxford, 1977.

[32] N. Lanfear and S. K. Suslov, The time-dependent Schrödinger equation, Riccati equation and Airy functions, arXiv:0903.3608v5 [math-ph] 22 Apr 2009.

[33] P. G. L. Leach, Berry's phase and wave functions for time-dependent Hamiltonian systems, J. Phys. A: Math. Gen 23 (1990), 2695-2699.

[34] P. G. L. Leach and K. Andriopoulos, The Ermakov equation: a commentary, Appl. Anal. Discrete Math. 2 (2008) \#2, 146-157.

[35] C. F. Lo, Coherent-state propagator of the generalized time-dependent parametric oscillator, Europhys. Lett. 24 (1993) \#5, 319-323.

[36] R. M. Lopez and S. K. Suslov, The Cauchy problem for a forced harmonic oscillator, Revista Mexicana de Física, 55 (2009) \#2, 195-215; see also arXiv:0707.1902v8 [math-ph] 27 Dec 2007.

[37] I. A. Malkin and V. I. Man'ko, Dynamical Symmetries and Coherent States of Quantum System, Nauka, Moscow, 1979 [in Russian].

[38] I. A. Malkin, V. I. Man'ko and D. A. Trifonov, Linear adiabatic invariants and coherent states, J. Math. Phys. 14 (1973) \#5, 576-582. 
[39] M. Meiler, R. Cordero-Soto, and S. K. Suslov, Solution of the Cauchy problem for a time-dependent Schrödinger equation, J. Math. Phys. 49 (2008) \#7, 072102: 1-27; see also arXiv: 0711.0559v4 [math-ph] 5 Dec 2007.

[40] S. Menouar, M. Maamache and J. R. Choi, An alternative approach to exact wave functions for time-dependent coupled oscillator model of charged particle in variable magnetic field, Ann. Phys., (2010) \#8, 1708-1719.

[41] E. Merzbacher, Quantum Mechanics, third edition, John Wiley \& Sons, New York, 1998.

[42] W Miller, Jr., Symmetry and Separation of Variables, Encyclopedia of Mathematics and Its Applications, Vol. 4, Addison-Wesley Publishing Company, Reading etc, 1977.

[43] A. F. Nikiforov, S. K. Suslov, and V. B. Uvarov, Classical Orthogonal Polynomials of a Discrete Variable, Springer-Verlag, Berlin, New York, 1991.

[44] V. M. Pérez-García, P. Torres and G. D. Montesinos, The method of moments for nonlinear Schrödinger equations: theory and applications, SIAM J. Appl. Math. 67 (2007) \#4, 990-1015.

[45] S. Rosencrans, Perturbation algebra of an elliptic operator, J. Math. Anal. Appl. 56 (1976) \#2, 317-329.

[46] E. Suazo and S. K. Suslov, Cauchy problem for Schrödinger equation with variable quadratic Hamiltonians, under preparation.

[47] E. Suazo and S. K. Suslov, Soliton-like solutions for nonlinear Schrödinger equation with variable quadratic Hamiltonians, arXiv:1010.2504 4 [math-ph] 24 Nov 2010.

[48] E. Suazo, S. K. Suslov and J. M. Vega-Guzmán, The Riccati equation and a diffusion-type equation, New York J. Math. 17a (2011), 225-244.

[49] E. Suazo, S. K. Suslov and J. M. Vega-Guzmán, The Riccati system and a diffusion-type equation, arXiv: 1102.4630v1 [math-ph] 22 Feb 2011.

[50] S. K. Suslov, Dynamical invariants for variable quadratic Hamiltonians, Physica Scripta 81 (2010) \#5, 055006 (11 pp); see also arXiv:1002.0144v6 [math-ph] 11 Mar 2010.

[51] S. K. Suslov, On integrability of nonautonomous nonlinear Schrödinger equations, to appear in Proc. Amer. Math. Soc.; see also arXiv:1012.3661v3 [math-ph] 16 Apr 2011.

[52] G. N. Watson, A Treatise on the Theory of Bessel Functions, Second Edition, Cambridge University Press, Cambridge, 1944.

[53] E. T. Whittaker and G. N. Watson, A Course of Modern Analysis, Fourth Edition, Cambridge University Press, Cambridge, 1927.

[54] K. B. Wolf, On time-dependent quadratic Hamiltonians, SIAM J. Appl. Math. 40 (1981) \#3, 419-431.

[55] K-H. Yeon, K-K. Lee, Ch-I. Um, T. F. George and L. N. Pandey, Exact quantum theory of a time-dependent bound Hamiltonian systems, Phys. Rev. A 48 (1993) \# 4, 2716-2720.

[56] A. V. Zhukov, Exact quantum theory of a time-dependent system with quadratic hamiltonian, Phys. Lett. A 256 (1999) \#5-6, 325-328.

School of Mathematical and Statistical Sciences, Arizona State University, Tempe, AZ 852871804, U.S.A.

E-mail address: nlanfear@asu.edu

Mathematical, Computational and Modeling Sciences Center, Arizona State University, Tempe, AZ 85287-1904, U.S.A.

E-mail address: rlopez14@asu.edu

School of Mathematical and Statistical Sciences \& Mathematical, Computational and Modeling Sciences Center, Arizona State University, Tempe, AZ 85287-1804, U.S.A.

E-mail address: sks@asu.edu

URL: http://hahn.la.asu.edu/ ${ }^{\sim}$ suslov/index.html 